\title{
Virtual bronchoscopic navigation combined with endobronchial ultrasound to diagnose small peripheral pulmonary lesions: a randomised trial
}

\author{
Takashi Ishida, ${ }^{1}$ Fumihiro Asano, ${ }^{2}$ Koichi Yamazaki, ${ }^{3}$ Naofumi Shinagawa, ${ }^{3}$ \\ Satoshi Oizumi, ${ }^{3}$ Hiroshi Moriya, ${ }^{4}$ Mitsuru Munakata, ${ }^{1}$ Masaharu Nishimura, ${ }^{3}$ for the \\ Virtual Navigation in Japan (V-NINJA) trial group
}

\section{See Editorial, p 1027 \\ ${ }^{1}$ Department of Pulmonary Medicine, Fukushima Medical University, Fukushima, Japan 2Department of Pulmonary Medicine, Gifu Prefectural General Medical Center, Gifu, Japan \\ ${ }^{3}$ First Department of Medicine, Hokkaido University School of Medicine, Sapporo, Japan ${ }^{4}$ Department of Radiology, Ohara General Hospital, Fukushima, Japan}

\section{Correspondence to}

Fumihiro Asano, Department of Pulmonary Medicine, Gifu

Prefectural General Medical Center, 4-6-1 Noishiki, Gifu 500-8717, Japan;

asano-fm@ceres.ocn.ne.jp

For author footnote see end of the article.

Received 29 June 2010 Accepted 16 June 2011 Published Online First 11 July 2011

\section{UNLOCKID}

This paper is freely available online under the BMJ Journals unlocked scheme, see http:// thorax.bmj.com/site/about/ unlocked.xhtml

\begin{abstract}
Background Bronchoscopy using endobronchial ultrasound (EBUS) can help to diagnose small peripheral pulmonary lesions. However, although biopsy sites can be confirmed, a bronchoscope cannot be guided in EBUS. Virtual bronchoscopic navigation (VBN) can guide a bronchoscope with virtual images, but its value has not been confirmed.
\end{abstract}

Methods This prospective multicentre study examines the value of VBN-assisted EBUS for diagnosing small peripheral pulmonary lesions. 199 patients with small peripheral pulmonary lesions (diameter $\leq 30 \mathrm{~mm}$ ) were randomly assigned to VBN-assisted (VBNA) or non-VBN-assisted (NVBNA) groups. A bronchoscope was introduced into the target bronchus of the VBNA group using the VBN system. Sites of specimen sampling were verified using EBUS with a guide sheath under fluoroscopy.

Results The diagnostic yield was higher for the VBNA than for the NVBNA group (80.4\% vs $67.0 \% ; p=0.032$ ). The duration of the examination and time elapsed until the start of sample collection were reduced in the VBNA compared with the NVBNA group (median (range), 24.0 $(8.7-47.0)$ vs $26.2(11.6-58.6) \mathrm{min}, \mathrm{p}=0.016)$ and 8.1 $(2.8-39.2)$ vs $9.8(2.3-42.3) \mathrm{min}, \mathrm{p}=0.045$, respectively). The only adverse event was mild pneumothorax in a patient from the NVBNA group. Conclusions The diagnostic yield for small peripheral pulmonary lesions is increased when VBN is combined with EBUS.

Clinical trial number UMIN000000569.

\section{INTRODUCTION}

Lung cancer is the leading cause of cancer death in Europe, the USA and Japan. ${ }^{1-3}$ Although imaging modalities including CT, MRI and positron emission tomography have been applied, pathological findings remain the benchmark for a diagnosis of lung cancer. The increased frequency of high quality CT application has allowed the identification of much smaller and far more pulmonary lesions than before. ${ }^{4}$

Suspected malignant pulmonary lesions can be diagnosed by bronchoscopy, but the sensitivity of detecting small peripheral lung cancer varies from $36 \%$ to $86 \%$ depending on the size of the lesion. ${ }^{5-7}$ According to the lung cancer diagnosis and treatment guidelines issued by the American College of

\section{Key messages}

What is the key question?

- Can virtual bronchoscopic navigation improve the bronchoscopic diagnostic yield for small peripheral pulmonary lesions?

What is the bottom line?

- Bronchoscopy using endobronchial ultrasound (EBUS) can help to diagnose peripheral pulmonary lesions; however, EBUS cannot navigate the bronchoscope itself, so small lesions cannot always be reached.

\section{Why read on?}

- This multicentre, randomised study shows that the diagnostic yield for small peripheral pulmonary lesions is increased when virtual bronchoscopic navigation is combined with EBUS.

Chest Physicians (ACCP), ${ }^{8}$ the diagnostic sensitivity of bronchoscopy for peripheral pulmonary lesions is $78 \%$, whereas that for lesions $<2 \mathrm{~cm}$ is $34 \%$. In comparison, the reported diagnostic sensitivity of transthoracic needle aspiration (TTNA) is $90 \%$, and TTNA is recommended for diagnosing lesions $<2 \mathrm{~cm}$. Consequently, TTNA is frequently applied in many countries, but the incidence of complications is fairly high. The British Thoracic Society (BTS) guidelines state that the incidence of pneumothorax is $0-61 \%$, with chest tube drainage required in $3.3-15 \%$, intrapulmonary haemorrhage in $5-16.9 \%$ and haemoptysis in $1.25-5 \%{ }^{9}$ The ACCP guidelines state with respect to bronchoscopy that, '... in expert hands, a radial probe US device can increase the diagnostic yield of $\mathrm{FB}$ while dealing with peripheral lesions of $<20 \mathrm{~mm}$ in size. Its use can be considered prior to referring the patient for TTNA.' The given grade of recommendation is $2 \mathrm{~B}$. ${ }^{8}$ Target lesions can be directly visualised by EBUS before attempting biopsies that raise the diagnostic yields for peripheral lesions ${ }^{10}$; reported diagnostic yields are $58.3-80 \% .{ }^{10-13}$ However, EBUS cannot navigate the bronchoscope itself, so lesions cannot be reached in $8-20.8 \%$ of cases. ${ }^{11} 13$

Navigational bronchoscopy has recently improved the diagnostic approach to peripheral small lesions. Electromagnetic navigation (EMN) is 
one of two methods that are currently in clinical use, and one EMN system (inReach System; superDimension, Minneapolis, Minnesota, USA) has been marketed mainly in Europe and in the USA. ${ }^{14}$ Bronchoscopists navigate EMN systems using a positional sensor that determines direction based on an electromagnetic field generated around the patient's chest. Reported diagnostic yields of EMN for variously sized peripheral pulmonary lesions range from $69 \%$ to $74 \%{ }^{15-17}$ A randomised study has shown that EMN-assisted bronchoscopy combined with EBUS is more sensitive than either modality alone (diagnostic yield, $88 \%$ vs $69 \%$ and $59 \%$, respectively). ${ }^{18}$

Virtual bronchoscopic navigation (VBN) is another method in which virtual bronchoscopic images of the bronchial path to a peripheral lesion are generated and used as a guide to navigate the bronchoscope. ${ }^{19}$ Since virtual bronchoscopic (VB) and bronchoscopic images are similar, the bronchoscope can be advanced near a target lesion according to the bronchial pathway displayed on the VB images. A VBN system has been developed that allows the automatic production of virtual images of the bronchial path that are matched with actual images for reliable bronchoscopic navigation to sampling sites. ${ }^{20}{ }^{21}$ However, the value of VBN has not yet been clearly and statistically demonstrated. The present multicentre, prospective, randomised study examines the value of VBN-assisted EBUS for diagnosing small peripheral pulmonary lesions of suspected lung cancer.

\section{METHODS \\ Participants}

We enrolled 199 patients who were referred to three Japanese medical centres between April 2006 and August 2007 with peripheral pulmonary lesions (mean diameter $\leq 3 \mathrm{~cm}$ calculated from axial CT images) suspected to be cancer that were not pathologically confirmed. Peripheral pulmonary lesions were defined as those that are surrounded by normal lung parenchyma and thus unlikely to be visualised by bronchoscopy. Most of these lesions were discovered by plain chest x-rays and/or CT images acquired for reasons other than the symptoms caused by the lesions. Eligible patients were men and women $\geq 20$ years old who could tolerate bronchoscopy. The exclusion criteria comprised evidence of endobronchial disease revealed by chest
CT, percutaneous oxygen saturation $<90 \%$, a range of known severe co-morbid conditions (unstable angina, acute myocardial infarction within the past 3 months, severe asthma or uncontrolled pulmonary infection), pregnancy and unable to proceed without anticoagulant or antiplatelet medications. We monitored the course of lesions that were $<10 \mathrm{~mm}$ with ground-glass opacity confirmed by CT and excluded them from the study.

\section{Randomisation and intervention}

Eligible patients were randomly assigned to VBN-assisted (VBNA) or non-VBN-assisted (NVBNA) groups. Because others have shown that bronchoscopic diagnostic yield is associated with lesion size $e^{8}$ and physician skill, randomisation was based on lesion size (mean diameter $<2 \mathrm{~cm}$ or $2-3 \mathrm{~cm}$ ) and bronchoscopists used a randomised block design to ensure that these factors were balanced in the study arms. Independent, blinded, trial staff randomly assigned the patients before bronchoscopy.

Scan data from multidetector chest CT (16- or 64-row; slice width, $0.5-2 \mathrm{~mm}$ ) were acquired from all patients without using contrast medium before bronchoscopy. Individual CT data sets from the VBNA group were transferred to a workstation on which VBN software automatically created virtual bronchoscopic images ${ }^{21}$ within $20 \mathrm{~min}$. The consecutive images could be moved forwards and backwards and rotated like a bronchoscope in a monitor positioned beside the video-bronchoscopic screen in the endoscopy suite. As assistant physician controlled the virtual bronchoscopic images ${ }^{20}$ during bronchoscopy and a bronchoscopist inserted an endoscope as instructed (figure 1). All patients were locally anaesthetised with lidocaine and examined using a thin video-bronchoscope (type P260F; outer diameter, $4.0 \mathrm{~mm}$; Olympus Medical Systems, Tokyo, Japan). Additional pentazocine hydrochloride or hydroxyzine chloride was administered as required.

Bronchoscopic insertion was assisted using the VBN system in the VBNA group. The bronchoscope was introduced into the bronchus of the NVBNA group without VBN support and with reference only to CT axial images. Lesions were visualised in both groups by inserting a $20 \mathrm{MHz}$ mechanical radial-type EBUS probe (external diameter, $1.4 \mathrm{~mm}$; UM-S20-17S; Olympus Medical Systems) with a guide sheath (K-201; Olympus Medical

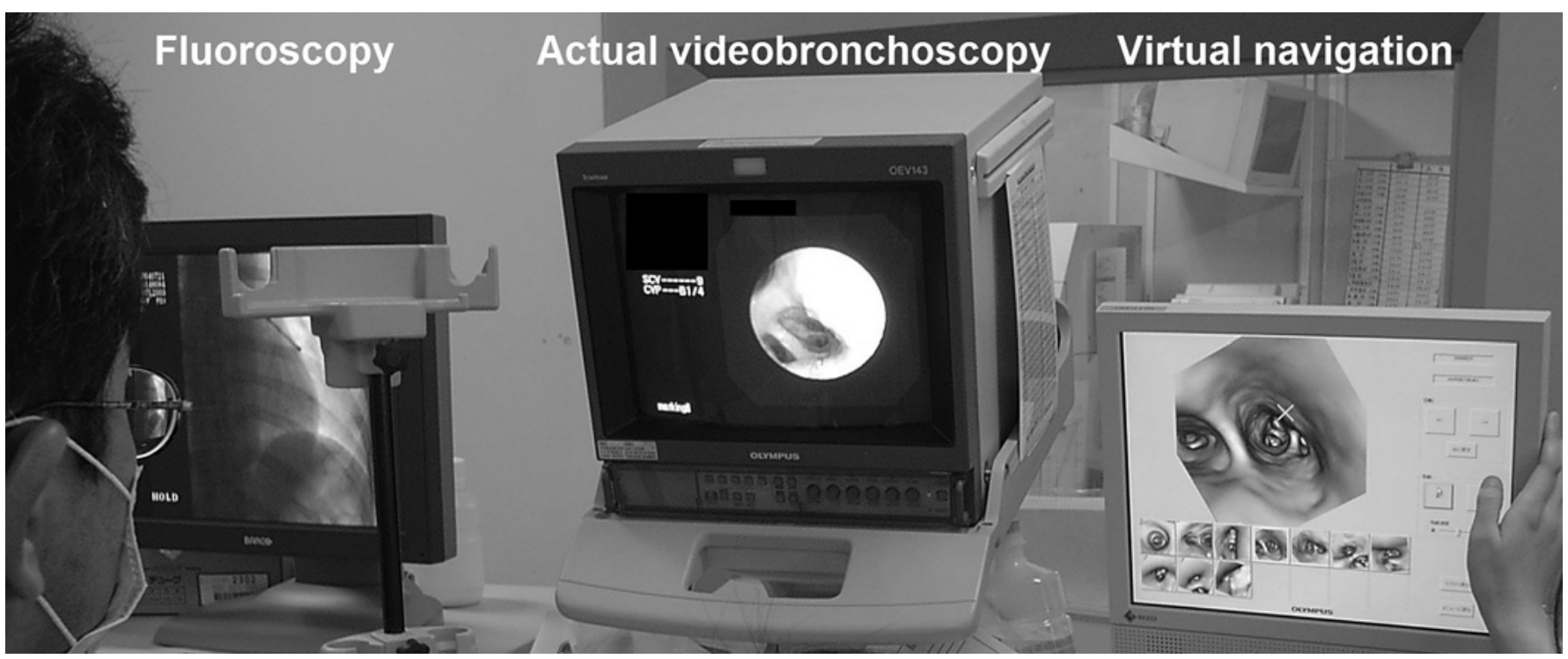

Figure 1 Virtual bronchoscopic navigation. An assistant physician controls virtual bronchoscopic images of the path leading to a peripheral lesion and a bronchoscopist inserts an endoscope as instructed. 
Systems) through an endoscopic channel. This probe was withdrawn as soon as the lesion was visualised. Pathological samples were collected using forceps and/or a brush introduced into the guide sheath. ${ }^{12}$ If the lesion was not visualised by EBUS, the approach to sampling was decided by the bronchoscopist. The area around the bronchial target was washed as determined by the bronchoscopist with $20 \mathrm{ml}$ of saline as a supplementary procedure. Biopsy samples were immediately fixed in formalin, brush smears on glass slides were immediately fixed in alcohol, and pathologists who were blinded to the results of randomisation processed and evaluated all specimens using standard procedures. The presence of bacteria was assessed in some portions of brush smears and/or lavage. All patients were positioned on an $\mathrm{x}$-ray table, correct device placement was confirmed and sampling was conducted under fluoroscopy.

\section{Outcomes}

The primary end point was bronchoscopic diagnostic yield defined as all instances in which the results matched the final diagnosis confirmed by pathological and/or bacterial assessment of bronchoscopic samples. The key secondary end point was total examination duration, which was calculated as the interval between the moment the endoscope passed the vocal cords until its withdrawal from the trachea. Other secondary end points were the interval until the start of sample collection, duration of $\mathrm{x}$-ray fluoroscopy and the generation number of the inserted bronchi. A segmental bronchus was defined as third generation. Safety end points of interest included severe haemorrhage, pneumothorax, hypoxaemia, lidocaine intoxication, arrhythmia, pneumonia and other serious adverse events. Retrieved blood loss $>50 \mathrm{ml}$ mixed with or without saline lavage was defined as significant. The safety of all patients was assessed.

\section{Study follow-up}

If a lesion was undiagnosed by bronchoscopy, we recommended that the patient consider undergoing other diagnostic procedures, including CT-guided fine needle aspiration (FNA) or surgical intervention. If an undiagnosed patient refused further intervention, follow-up was considered as the second best strategy. Thus, this study continued until the last enrolled patient had been followed up for 2 years. Follow-up information was derived from outpatient clinics, by telephone or by fax contact.

\section{Statistical analysis}

Sample size was calculated based on the primary end point. The estimated diagnostic yields in the VBNA and NVBNA groups based on published records were $70 \%$ and $50 \%$, respectively. ${ }^{13} 2122$ Thus, at $80 \%$ power and $\alpha=0.05$, we calculated that 190 patients would be required ( $\mathrm{n}=95$ in each group) to determine whether diagnostic yield improved with the addition of VBNA. We planned to enrol 200 patients to account for incomplete data or undiagnosed patients.

We analysed the diagnostic yield and safety of the entire intent-to-treat population. Data from the per-protocol population that included all randomised patients with planned bronchoscopic procedures for peripheral lesions were also statistically analysed. Primary and secondary variables were analysed using
Figure 2 CONSORT flow diagram. *Al allocated patients were included in the intention-to-treat population. †Diagnoses established without operating virtual bronchoscopic navigation (VBN). ‡The bronchoscopic procedure was performed according to protocol. §Final diagnosis of this patient was not established; however, this patient was included in the per-protocol analysis.

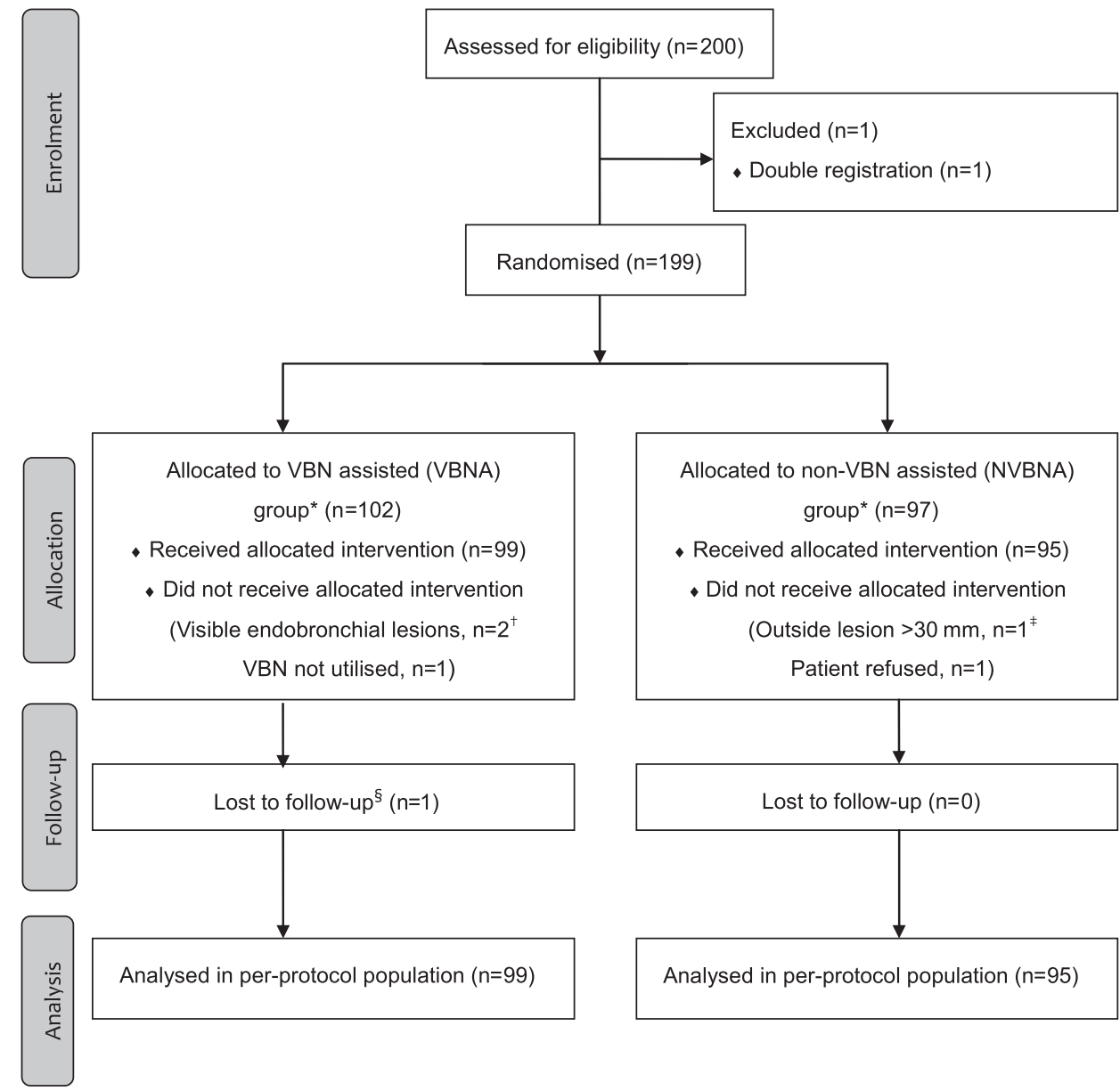


the Pearson $\chi^{2}$ test and the Mann-Whitney U test. Continuous variables were assessed for normality in distribution and the Mann-Whitney test/description as medians was used. All $p$ values were two-sided. A $p$ value of $<0.05$ indicated statistical significance. All data were statistically analysed using IBM SPSS Statistics, V 18.

\section{RESULTS}

We randomly assigned 102 and 97 patients to the VBNA and NVBNA groups. One and two patients in the NVBNA and VBNA groups, respectively, with endobronchially visible malignant or large $(>3 \mathrm{~cm})$ lesions were ineligible. We could not use $\mathrm{VBN}$ in one patient in the VBNA group and another refused bronchoscopy after assignment to the NVBNA group. We excluded these five patients from the per-protocol populations. The trial profile is shown in figure 2. Age, sex, lesion size and location in patients at baseline were similar between the groups (table 1)

The proportions of primary lung cancer, other malignancies and non-malignant diseases were similar between the groups. Non-malignant diseases comprised tuberculosis, non-tuberculous mycobacterial infection, fungal disease, organising pneumonia, hamartoma and lipoma. Among bronchoscopically undiagnosed patients, 23 (44.2\%) of 52 underwent video-assisted thoracoscopy and/or surgery and 8 (15.4\%) were diagnosed by CT-guided FNA or repeated bronchoscopy. Twenty of the 21 patients who refused further intervention were followed up for 2 years.

The results indicated a significant difference in diagnostic yield between arms both in the intent-to-treat (all randomly assigned patients, $\mathrm{n}=199$ ) and the per-protocol populations (finally analysed patients, $n=194$ ). Diagnostic yield was significantly higher for the VBNA than for the NVBNA groups in both the intent-to-treat (82 (80.4\%) of 102 vs $65(67.0 \%)$ of 97; $\mathrm{p}=0.032)$ and per-protocol (80 (80.8\%) of 99 vs $64(67.4 \%)$ of 95 ; $\mathrm{p}=0.032$; table 2) populations.

Diagnostic yield did not differ significantly according to lesion size between the groups in the per-protocol population (table 3).

Table 1 Baseline characteristics and final diagnoses

\begin{tabular}{|c|c|c|}
\hline & $\begin{array}{l}\text { VBNA group } \\
(n=102)\end{array}$ & $\begin{array}{l}\text { NVBNA group } \\
(\mathrm{n}=97)\end{array}$ \\
\hline Age (years, median; range) & $69(21-85)$ & $67(27-82)$ \\
\hline Male, n (\%) & $64(62.7)$ & $57(58.8)$ \\
\hline Lesion size (mm, median; range) & $18.0(9.5-30.0)$ & $18.0(7.0-30.0)$ \\
\hline$<20 \mathrm{~mm}, \mathrm{n}(\%)$ & $59(57.8)$ & $59(60.8)$ \\
\hline $20-30$ mm, n (\%) & $43(42.2)$ & $38(39.2)$ \\
\hline \multicolumn{3}{|l|}{ Lesion location } \\
\hline Rt. upper lobe, n (\%) & $32(31.4)$ & $35(36.1)$ \\
\hline Rt. middle lobe, $\mathrm{n}(\%)$ & $12(11.8)$ & $6(6.2)$ \\
\hline Rt. lower lobe, n (\%) & $23(22.5)$ & $18(18.6)$ \\
\hline Lt. upper lobe, n (\%) & $25(24.5)$ & $21(21.6)$ \\
\hline Lt. lower lobe, n (\%) & $10(9.8)$ & $17(17.5)$ \\
\hline \multicolumn{3}{|l|}{ Final diagnosis } \\
\hline \multicolumn{3}{|l|}{ Malignant disease $\mathrm{n}(\%)$} \\
\hline Primary lung cancer $\mathrm{n}(\%)$ & $69(67.6)$ & $76(78.4)$ \\
\hline Other malignant disease $\mathrm{n}(\%)$ & $10(9.8)$ & $4(4.1)$ \\
\hline \multicolumn{3}{|l|}{ Non-malignant disease $\mathrm{n}(\%)$} \\
\hline Infectious disease $\mathrm{n}(\%)$ & $17(16.7)$ & $8(8.2)$ \\
\hline Other benign condition $\mathrm{n}(\%)$ & $5(4.9)$ & $9(9.3)$ \\
\hline Undetermined $\mathrm{n}(\%)$ & $1(1.0)$ & $0(0)$ \\
\hline
\end{tabular}

Lt., left; NVBNA, non-virtual bronchoscopic navigation-assisted; Rt., right; VBNA, virtual bronchoscopic navigation-assisted.
Table 2 Diagnostic yields in the intent-to-treat and per-protocol populations

\begin{tabular}{lrrr}
\hline & \multicolumn{2}{l}{ Bronchoscopic diagnosis } & \\
\cline { 2 - 3 } & VBNA & p Value \\
\hline Full intent-to-treat & $82 / 102(80.4)$ & $65 / 97(67.0)$ & 0.032 \\
Per-protocol & $80 / 99(80.8)$ & $64 / 95(67.4)$ & 0.032 \\
\hline
\end{tabular}

Data are shown as numbers of lesions/total lesions (\%).

NVBNA, non-virtual bronchoscopic navigation-assisted; VBNA, virtual bronchoscopic navigation-assisted.

Of the virtual images constructed based on data from the VBNA group, 98\% agreed with actual images of the shape of each bronchial bifurcation on the route. The median generation of virtual bronchial images was six (range, 4-12 bronchi).

The VBN system thus allowed insertion of the endoscope into significantly further generations of bronchi (VBNA vs NVBNA median (range): 4 (2-8) vs $4(2-7) ; p<0.001 ;$ table 4$)$. The endoscope was also accurately positioned in the VBNA group, with more targets being confirmed by EBUS (VBNA vs NVBNA: $92 / 99$ (92.9\%) vs $77 / 95$ (81.1\%); $p=0.014)$. Numbers of biopsies and brushings/washes did not differ significantly between the groups. Total examination duration was significantly shorter in the VBNA than in the NVBNA group (median (range): 24.0 (8.7-47.0) vs $26.2(11.6-58.6) \mathrm{min} ; \mathrm{p}=0.016)$. The interval to starting sample collection was significantly shorter in the VBNA versus the NVBNA group (8.1 (2.8-39.2) vs $(9.8$ (2.3-42.3) min; $\mathrm{p}=0.045)$. The duration of $\mathrm{x}$-ray fluoroscopy exposure did not differ significantly between the groups. No severe or moderate adverse events were associated with bronchoscopy except for mild pneumothorax that did not require chest drainage in a patient from the NVBNA group.

\section{DISCUSSION}

This is the first prospective, multicentre, randomised trial to examine the value of using a VBN system to assist radial EBUS. The findings showed that VBN-assisted EBUS with a guide sheath significantly improved the diagnostic yield of small pulmonary peripheral lesions to $80.4 \%$, which was $13 \%$ higher than that in the NVBNA group (67.0\%). The diagnostic yield for similar lesions in our previous study using a conventional or thin bronchoscope assisted by VBN under fluoroscopy was $62.5 \%{ }^{23}$ The diagnostic yield was $58.3 \%$ in another of our studies using EBUS. ${ }^{13}$ The high diagnostic yield in the present study was achieved by combining these two procedures. Moreover, although the study cohort was small and diagnostic yields for lesions $<20 \mathrm{~mm}$ did not differ significantly, the diagnostic yield with VBNA was nevertheless high at $75.9 \%$. Comparison is difficult since $x$-ray fluoroscopy was used in this study, but the diagnostic yield with EBUS + VBN was comparable with that reported by Eberhardt et al. ${ }^{18}$ One advantage of VBN compared with EMN is simplicity. Operations such as steering the sensor

Table 3 Diagnostic yield according to lesion size in the per-protocol population

\begin{tabular}{llll}
\hline \multirow{2}{*}{ Lesion size } & \multicolumn{2}{l}{ Bronchoscopic diagnosis } & \\
\cline { 2 - 3 } & VBNA & NVBNA & p Value \\
\hline$<20 \mathrm{~mm}$ & $44 / 58(75.9)$ & $35 / 59(59.3)$ & 0.056 \\
$20-30 \mathrm{~mm}$ & $36 / 41(87.8)$ & $29 / 36(80.6)$ & 0.382 \\
Total & $80 / 99(80.8)$ & $64 / 95(67.4)$ & 0.032 \\
\hline
\end{tabular}

Data are presented as numbers of lesions/total lesions (\%)

NVBNA, non-virtual bronchoscopic navigation-assisted; VBNA, virtual bronchoscopic navigation-assisted. 
Table 4 Bronchoscopic outcomes in the per-protocol population

\begin{tabular}{lccc}
\hline & VBNA & NVBNA & p Value \\
\hline $\begin{array}{l}\text { Endoscopically inserted bronchial } \\
\text { generation (n, median) (range) }\end{array}$ & $4(2-8)$ & $4(2-7)$ & $<0.001$ \\
$\begin{array}{l}\text { EBUS-visualised peripheral } \\
\text { lesion, n (\%) }\end{array}$ & $92(92.9)$ & $77(81.1)$ & 0.014 \\
$\begin{array}{l}\text { Sampling by biopsy, (n, median) } \\
\text { (range) }\end{array}$ & $5(0-12)$ & $4(0-12)$ & 0.113 \\
$\begin{array}{l}\text { Sampling by brushing/washing } \\
\text { (n, median) (range) }\end{array}$ & $3(0-6)$ & $3(0-5)$ & 0.42 \\
$\begin{array}{l}\text { Duration } \\
\begin{array}{l}\text { Total examination (min, median) } \\
\text { (range) }\end{array}\end{array}$ & $24.0(8.7-47.0)$ & $26.2(11.6-58.6)$ & 0.016 \\
$\begin{array}{l}\text { Initial sampling (min, median) } \\
\text { (range) }\end{array}$ & $8.1(2.8-39.2)$ & $9.8(2.3-42.3)$ & 0.045 \\
$\begin{array}{l}\text { x-ray fluoroscopy exposure } \\
\text { (min, median) (range) }\end{array}$ & $9.7(1.5-22.7)$ & $11.0(1.3-31.0)$ & 0.058 \\
\hline
\end{tabular}

EBUS, endobronchial ultrasound; NVBNA, non-virtual bronchoscopic navigation-assisted; VBNA, virtual bronchoscopic navigation-assisted.

probe or registration during tests are not required to superimpose the electromagnetic sensor position and CT information as with EMN. ${ }^{14}$ Furthermore, the bronchoscope is advanced together with virtual image indications in VBN, which is essentially identical to conventional bronchoscope manipulation. ${ }^{19}$ Thus, bronchoscopists with basic skills can easily operate this system after practice on a simulator. Locatable guides attached to the EMN sensor probe are single use, which imposes a cost burden of US\$700-1000 per patient depending on market price. $^{24}$

The diagnostic sensitivity of TTNA differs depending on the technique and lesion size, but it is $92 \%$ with FNA under CT guidance $^{6}$ and $82-90 \%$ even for lesions $\leq 2 \mathrm{~cm}$. However, the main complication with FNA under CT guidance is pneumothorax, which occurs at an incidence of $15-42 \%^{25-27}$ and is quite frequent with small lesions or emphysema. ${ }^{27}$ Reported complications comprise bronchial haemorrhage, ${ }^{25}$ needle tract implantation and air embolism. ${ }^{28}$ In contrast, the complication rate with transbronchial lung biopsy is $0.2-5 \%$ for pneumothorax and 1.2-9\% for haemorrhage. ${ }^{9} 2930$ Pneumothorax or other complications have not been found in other studies of EBUS + VBN to date, including the present study. ${ }^{21} 22$ The diagnostic sensitivity of EBUS + VBN may not be as high as that with CT-guided FNA, but it is considerably higher than the diagnostic sensitivity of normal bronchoscopy indicated in the ACCP guidelines. ${ }^{8}$ Therefore, considering the low rate of complications, VBN combined with EBUS may be a viable option for diagnosing small peripheral lesions.

To our knowledge, this is the first report to describe the duration of navigational bronchoscopy, and that the amount of time required for guidance was decreased in the VBNA group. Moreover, VBN improved diagnostic yield while decreasing the overall duration of the examination by $\sim 2 \mathrm{~min}$. This is thought to be significant in terms of patient comfort, especially considering that patients endured the procedure under local anaesthesia. Although the time required for fluoroscopy tended to decrease in the VBNA group, the difference did not reach statistical significance. Fluoroscopy duration before and after initial sampling was not measured in this study, but almost all of the fluoroscopic exposure was taken up by specimen sampling to confirm proper device use, such as forceps opening and cutting, as well as brushing at adequate sites. Steinfort et al reported that radiation exposure from fluoroscopy used together with EBUS does not pose a clinical problem. ${ }^{31}$ However, we found that radiation exposure accounted for $\sim 40 \%$ of the total duration of the examination. More reliable sampling devices are needed for EBUS with a guide sheath.

We identified the following limitations. The precision of $\mathrm{VB}$ decreases when CT data are inadequate during VB; for example, the branch order that can be visualised is lower when slices are too thick. ${ }^{32}$ Under such circumstances, repeat CT might be required, which imposes additional cost. Many of the patients in this study were referred from private clinics after abnormalities on plain x-rays had been identified. Therefore, row CT data with a slice thickness of $\leq 2 \mathrm{~mm}$ were collected from the start with multidetector CT instruments at the institution where the present study was conducted and, as a result, virtual images could be created up to sixth generation bronchi. In contrast, the amount of CT data increases with thinner slices and more time is required to create $\mathrm{VB}$ images. Most of the time required to create $\mathrm{VB}$ images in this study was due to $\mathrm{PC}$ processing, but the $\mathrm{VB}$ images were created over a period of $\sim 20 \mathrm{~min}$. This depends to some extent on the performance of the software and PC hardware, which could be further enhanced. The VBN system used in the present study (Bf-NAVI; Cybernet Systems, Tokyo, Japan) has been promoted mainly in Japan. The automatic tracking system for the VBN system used herein will allow automatic synchronisation of the virtual to the actual bronchoscopic view. ${ }^{33}$ Another VBN system with an automatic tracking system has recently been developed. $^{24} 34$ This study showed that diagnostic yield improved with VBN when combined with EBUS which is still not globally applied. We also combined VBN with fluoroscopy, and confirmed lesions in $83 \%$ of patients with plain x-rays. Although VBN has been applied without fluoroscopy, ${ }^{24}$ when not combined with EBUS it is usually combined with $\mathrm{CT}^{20} 35$ or fluoroscopy ${ }^{23}$ to confirm lesions. Randomised studies with each of these combinations may be necessary to define the diagnostic value of $\mathrm{VBN}$.

\section{Author footnote}

The V-NINJA Investigators: Principal investigator: Fumihiro Asano (Department of Pulmonary Medicine, Gifu Prefectural General Medical Center, Gifu, Japan).

Investigators: Koichi Yamazaki, Naofumi Shinagawa, Satoshi Oizumi, Eiki Kikuchi, Hajime Asahina, Noriyuki Yamada, Hiroshi Yokouchi, Chie Yoshida, Masaharu Nishimura (First Department of Medicine, Hokkaido University School of Medicine, Sapporo, Japan): Yuya Onodera, Kazuo Miyasaka (Department of Radiology, Hokkaido University School of Medicine, Sapporo, Japan); Yoshihiko Matsuno, Akifumi Tsuzuku Masaki Anzai, Atsunori Masuda (Department of Pulmonary Medicine, Gifu Prefectural General Medical Center, Gifu, Japan); Hiroshi Moriya (Department of Radiology, Ohara General Hospital, Fukushima, Japan); Takashi Ishida, Motoko Tachihara, Kenya Kanazawa, Aya Sugawara, Kana Watanabe, Kumi Uekita, Kengo Oshima, Satoko Sekine, Mitsuru Munakata (Department of Pulmonary Medicine, Fukushima Medical University, Fukushima, Japan).

Acknowledgements We thank all the physicians and endoscopy suite personne involved in this trial for their cooperation and recruitment of patients. The authors and participants in this study wish to dedicate this manuscript, when published, to the memory of the former principal investigator in this study, Dr Koichi Yamazaki, who passed away on 12 January 2008

Funding This study was supported by funding from the Faculty of Medicine, Hokkaido University School of Medicine. During the trial period, VBN software-installed workstations were provisionally provided according to a contract with Olympus Medical Systems Corporation, Tokyo, Japan. The funding source and Olympus Medical Systems Corporation did not participate in the design and implementation of the study or data analysis, in writing the manuscript or in the decision to publish.

Competing interests FA, HM, KY, TI and Olympus Medical Systems Corporation co-developed the VBN system. FA, HM, KY and Tl legally transferred all patent rights to Olympus Corporation without compensation. TI, FA and NS have received speaker fees of less than three hundred thousand yen ( US $\$ 3500$ ) per year each from the Olympus Corporation as invited guests to academic medical meetings. All other authors declare that they have no conflict of interest.

Patient consent Obtained. 
Ethics approval This study was conducted with the approval of the ethics committee of each of the following institutions: Gifu Prefectural General Medical Center, Gifu, Japan, Hokkaido University School of Medicine, Sapporo, Japan and Fukushima Medical University, Fukushima, Japan.

Provenance and peer review Not commissioned; externally peer reviewed.

\section{REFERENCES}

1. Sant M, Allemani C, Santaquilani M, et al. EUROCARE-4. Survival of cancer patients diagnosed in 1995-1999. Results and commentary. Eur J Cancer 2009;45:931-91.

2. Jemal A, Siegel R, Ward E, et al. Cancer statistics, 2009. CA Cancer J Clin 2009;59:225-49.

3. Matsuda T, Marugame T, Kamo K, et al. Cancer incidence and incidence rates in Japan in 2002: based on data from 11 population-based cancer registries. Jpn J Clin Oncol 2008;38:641-8.

4. Jacobs PC, Mali WP, Grobbee DE, et al. Prevalence of incidental findings in computed tomographic screening of the chest: a systematic review. J Comput Assist Tomogr 2008; 32:214-21.

5. Baaklini WA, Reinoso MA, Gorin $A B$, et al. Diagnostic yield of fiberoptic bronchoscopy in evaluating solitary pulmonary nodules. Chest 2000;117:1049-54.

6. Schreiber G, McCrory DC. Performance characteristics of different modalities for diagnosis of suspected lung cancer: summary of published evidence. Chest 2003;123:115S-28S

7. Yung RC. Tissue diagnosis of suspected lung cancer: selecting between bronchoscopy, transthoracic needle aspiration, and resectional biopsy. Respir Care Clin N Am 2003;9:51-76

8. Rivera MP, Mehta AC. Initial diagnosis of lung cancer: ACCP evidence-based clinical practice guidelines (2nd edition). Chest 2007;132(3 Suppl):131S-48S

9. Manhire A, Charig M, Clelland C, et al. Guidelines for radiologically guided lung biopsy. Thorax 2003;58:920-36.

10. Paone G, Nicastri E, Lucantoni G, et al. Endobronchial ultrasound-driven biopsy in the diagnosis of peripheral lung lesions. Chest 2005:128:3551-7.

11. Herth FJ, Ernst A, Becker HD. Endobronchial ultrasound-guided transbronchial lung biopsy in solitary pulmonary nodules and peripheral lesions. Eur Respir J 2002;20:972-4.

12. Kurimoto N, Miyazawa T, Okimasa S, et al. Endobronchial ultrasonography using a guide sheath increases the ability to diagnose peripheral pulmonary lesions endoscopically. Chest 2004;126:959-65.

13. Kikuchi E, Yamazaki K, Sukoh N, et al. Endobronchial ultrasonography with guidesheath for peripheral pulmonary lesions. Eur Respir J 2004;24:533-7.

14. Schwarz Y, Mehta AC, Ernst A, et al. Electromagnetic navigation during flexible bronchoscopy. Respiration 2003;70:516-22.

15. Becker H, Herth F, Ernst A. Bronchoscopic biopsy of peripheral lung lesions under electromanetic guidance: a pilot study. J Bronchol 2005:12:9-13.

16. Schwarz Y, Greif J, Becker HD, et al. Real-time electromagnetic navigation bronchoscopy to peripheral lung lesions using overlaid CT images: the first human study. Chest 2006:129:988-94.

17. Gildea TR, Mazzone PJ, Karnak D, et al. Electromagnetic navigation diagnostic bronchoscopy: a prospective study. Am J Respir Crit Care Med 2006:174:982-9.
18. Eberhardt R, Anantham D, Ernst A, et al. Multimodality bronchoscopic diagnosis of peripheral lung lesions: a randomized controlled trial. Am J Respir Crit Care Med 2007;176:36-41.

19. Asano F, Matsuno $Y$, Matsushita $T$, et al. Transbronchial diagnosis of a pulmonary peripheral small lesion using an ultrathin bronchoscope with virtual bronchoscopic navigation. J Bronchol 2002:9:108-11.

20. Asano F, Matsuno $Y$, Shinagawa N, et al. A virtual bronchoscopic navigation system for pulmonary peripheral lesions. Chest 2006;130:559-66.

21. Asano F, Matsuno $Y$, Tsuzuku A, et al. Diagnosis of peripheral pulmonary lesions using a bronchoscope insertion guidance system combined with endobronchial ultrasonography with a guide sheath. Lung Cancer 2008;60:366-73.

22. Asahina H, Yamazaki K, Onodera Y, et al. Transbronchial biopsy using endobronchia ultrasonography with a guide sheath and virtual bronchoscopic navigation. Chest 2005;128:1761-5.

23. Tachihara $\mathbf{M}$, Ishida $T$, Kanazawa $\mathrm{K}$, et al. A virtual bronchoscopic navigation system under X-ray fluoroscopy for transbronchial diagnosis of small periphera pulmonary lesions. Lung Cancer 2007:57:322-7.

24. Eberhardt R, Kahn N, Gompelmann D, et al. LungPoint-a new approach to peripheral lesions. J Thorac Oncol 2010;5:1559-63.

25. Laurent $\mathbf{F}$, Latrabe V, Vergier B, et al. CT-guided transthoracic needle biopsy of pulmonary nodules smaller than $20 \mathrm{~mm}$ : results with an automated 20-gauge coaxia cutting needle. Clin Radiol 2000;55:281-7.

26. Ohno Y, Hatabu H, Takenaka D, et al. CT-guided transthoracic needle aspiration biopsy of small $(<$ or $=20 \mathrm{~mm})$ solitary pulmonary nodules. AJR Am J Roentgenol 2003;180:1665-9.

27. Cox JE, Chiles C, McManus CM, et al. Transthoracic needle aspiration biopsy: variables that affect risk of pneumothorax. Radiology 1999;212:165-8.

28. Ibukuro K, Tanaka R, Takeguchi $\mathrm{T}$, et al. Air embolism and needle track implantation complicating CT-guided percutaneous thoracic biopsy: single-institution experience. AJR Am J Roentgenol 2009;193:W430-6.

29. Gasparini S, Ferretti M, Secchi EB, et al. Integration of transbronchial and percutaneous approach in the diagnosis of peripheral pulmonary nodules or masses. Experience with 1,027 consecutive cases. Chest 1995;108: 131-7.

30. Niwa H, Tanahashi M, Kondo T, et al. Bronchoscopy in Japan: a survey by the Japan Society for Respiratory Endoscopy in 2006. Respirology 2009;14:282-9.

31. Steinfort DP, Einsiedel P, Irving LB. Radiation dose to patients and clinicians during fluoroscopically-guided biopsy of peripheral pulmonary lesions. Respir Care 2010;55:1469-74.

32. Neumann K, Winterer J, Kimmig M, et al. Real-time interactive virtual endoscopy of the tracheo-bronchial system: influence of CT imaging protocols and observer ability. Eur J Radiol 2000;33:50-4.

33. Deguchi D, Akiyama K, Mori K, et al. A method for bronchoscope tracking by combining a position sensor and image registration. Comput Aided Surg 2006:11:109-17

34. Merritt SA, Gibbs JD, Yu KC, et al. Image-guided bronchoscopy for peripheral lung lesions: a phantom study. Chest 2008;134:1017-26.

35. Shinagawa N, Yamazaki K, Onodera Y, et al. CT-guided transbronchial biopsy using an ultrathin bronchoscope with virtual bronchoscopic navigation. Chest 2004:125:1138-43.

\section{Thorax online}

Visit Thorax online and listen to the latest podcast, post comments and download any you might have missed. Keep informed and up to date by visiting thorax.bmj.com. 